\title{
Psoriasis: Bei manchen Biologika muss man mit Infektionen rechnen
}

\begin{abstract}
Aus der prospektiven Beobachtungsstudie PSOLAR geht hervor, dass während der Psoriasistherapie die Wahrscheinlichkeit für schwere Infektionen bei Therapie mit den derzeit gebräuchlichen Biologika unterschiedlich hoch ist, und zwar im Vergleich zum Infektionsrisiko unter einer systemischen Behandlung mit Nichtbiologika.
\end{abstract}

\section{Hier steht eine Anzeige.}

In der Beobachtungsstudie PSOLAR („Psoriasis Longitudinal Assessment and Registry”) wird die Sicherheit und Wirksamkeit der Therapie von Psoriasispatienten untersucht, wobei das gesamte derzeit verfügbare Arzneispektrum berücksichtigt wird. Bei den Biologika handelt es sich um Ustekinumab (3.474 Patienten), Infliximab (1.151 Patienten), Adalimumab (2.675 Patienten) und Etanercept (1.854 Patienten). Unter den Medikamenten, die nicht gentechnisch hergestellt sind, befinden sich Kortikosteroide, Ciclosporin und Methotrexat.

Das Register, das im Juni 2007 eröffnet worden war, wurde über einen Zeitraum von nun acht Jahren mit Daten gefüttert. Bis 2013 wurden insgesamt 323 schwere Infektionen dokumentiert. Von diesen bewerteten die Ärzte 287 (88,9\%) deshalb als "schwer“, weil sie Grund für eine stationäre Einweisung waren oder den Klinikaufenthalt verlängerten. Das entspricht einer kumulativen Inzidenz von 1,45 pro 100 Patientenjahren. Bezogen auf die Gesamtpopulation der Studienteilnehmer betrug die Rate schwerer Infektionen unter Ustekinumab 0,83, Etanercept 1,47, Adalimumab 1,97 und Infliximab 2,49 pro 100 Patientenjahre. In der Gruppe der Patienten ohne Biologika und ohne Methotrexat lag der Wert bei 1,05 und in der Gruppe ohne Biologika, aber mit Methotrexat bei 1,28 pro 100 Patientenjahre.

Die häufigsten Infektionen waren Pneumonien und Zellulitis. Wie erwartet, seien Alter, Diabetes, Infektionen in der Anamnese sowie Rauchen signifikant mit schweren Infektionen assoziiert gewesen. Keine Assoziation gab es dagegen zwischen Psoriasisarthritis und schweren Infektionen.

Fazit: Die Studie bestätige, dass die Anwendung von Adalimumab und Infliximab im Vergleich zu Nichtbiologika (mit oder ohne Methotrexat) mit einem erhöhten Risiko für schwere Infektionen assoziiert sei, so die Autoren. Dagegen sei die Anwendung von Ustekinumab nicht mit einem erhöhten Risiko assoziiert, ebenfalls im Vergleich zur Therapie mit Nichtbiologika. Dies gelte auch für Etanercept. Bei der Interpretation der Ergebnisse ist allerdings zu berücksichtigen, dass es sich nicht um eine randomisierte, sondern nur um eine Beobachtungsstudie handelt und somit Verzerrungen bei der Therapiewahl und der Entscheidung für die freiwillige Patiententeilnahme aufgetreten sein können. Zudem bedeute statistische Signifikanz in dieser Studie nicht zugleich klinische Relevanz.

Peter Leiner

Kalb RE et al. Risk of Serious Infection With Biologic and Systemic

Treatment of Psoriasis: Results From the Psoriasis Longitudinal Assessment and Registry (PSOLAR). JAMA Dermatol 2015; 151: 961-9 